\title{
Treatment of Melasma Using a Novel 1,927 nm Fractional Thulium Laser: A Retrospective Analysis of 68 Korean Patients
}

Nark-Kyoung Rho

Leaders Aesthetic Laser \& Cosmetic Surgery Center, Seoul, Korea

Received May 22, 2017

Accepted May 27, 2017

\footnotetext{
Correspondence

Nark-Kyoung Rho

Leaders Aesthetic Laser \& Cosmetic Surgery Center, THE CLASSIC500 Building, 90

Neungdong-ro, Gwangjin-gu, Seoul 05065, Korea Tel.: +82-2-444-7585

Fax: +82-2-444-7535

E-mail: rhonarkahanmail.net

(C) Korean Society for Laser Medicine and Surgery

(c) This is an open access article distributed under the terms of the Creative Commons Attribution NonCommercial License (http://creativecommons.org/ licenses/by-nc/4.0) which permits unrestricted noncommercial use, distribution, and reproduction in any medium, provided the original work is properly cited.
}

\author{
Background and Objectives \\ To determine the efficacy and safety of a novel 1,927 nm fractional \\ thulium laser system in treating moderate-to-severe melasma in Korean \\ subjects.
}

\begin{abstract}
Materials and Methods
This study is a retrospective chart and photographic review of 68 Korean women (Fitzpatrick skin type III-IV, mean age of $43.9 \mathrm{yr}$ ) with a clinical diagnosis of melasma treated with a novel 1,927 nm fractional thulium laser system. Two independent and blinded investigators evaluated the Melasma Area Severity Index (MASI) scores for each patient, based on clinical photography.
\end{abstract}

\section{Results}

A total of 204 consecutive treatment sessions in 68 patients were included in the analysis. The mean MASI scores decreased from $10.7 \pm$ 5.3 to $7.6 \pm 4.6$, at 2 weeks after 3 consecutive laser treatments ( $p=0.0001)$. The subjective assessment of patients revealed that 33 out of 68 subjects were successful in clearing more than $50 \%$ of their melasma. Mild and transient postinflammatory hyperpigmentation developed in three patients. Two patients experienced worsening of pre-existing acne. Sixty patients were successfully followed-up (mean 4.1 months), and the recurrence rate was $21.7 \%$.

\section{Conclusion}

The novel 1,927 $\mathrm{nm}$ fractional thulium laser resurfacing appears to be safe and effective for melasma treatment in the Korean population.

\section{Key words}

Asian; Fractional laser; Korean; Melasma; Thulium laser 


\section{INTRODUCTION}

Melasma is a common acquired hypermelanosis characterized by irregular light to dark brown macules and patches on sun-exposed areas of the skin. ${ }^{1}$ Although its populational incidence is not precisely known, ${ }^{2}$ melasma is regarded as one of the most common pigmentary disturbances in adult Korean females. ${ }^{3}$ Sun exposure and pregnancy are suggested as the most common triggering or aggravating factors of melasma. ${ }^{1-3}$ According to a recent study of 400 Korean women with melasma, $61.1 \%$ of melasma patients complained of sensitive/inflammatory features such as erythema, itching, and a stinging sensation, ${ }^{4}$ which implies that melasma could be related with skin barrier disruption and/or chronic subclinical skin inflammation. Concomitant pigmentary disorders including post-inflammatory hyperpigmentation (PIH), pigmented contact dermatitis, and acquired bilateral nevus of Otalike macules are commonly associated with melasma. ${ }^{4}$

The histopathologic features of melasma are fully described in the literature. ${ }^{3}$ The amount of melanin is significantly increased in all epidermal layers and melanosomes are higher in number and more widely dispersed in the keratinocytes of melasma lesional skin of. In addition to epidermal pigmentation, the pathologic findings of melasma include extracellular matrix abnormalities, especially solar elastosis, ${ }^{5}$ suggesting that melasma could be a result of a cumulative sun exposure, in a microenvironment of cutaneous photoaging in which inflammatory cells play a key role. ${ }^{6}$ The disrupted basement membrane and an increase in vascularity have been described in melasma with variable incidences and this indicates that dermal factors have critical roles in the pathogenesis of melasma, despite the fact that melasma is characterized by epidermal hyperpigmentation. ${ }^{5}$ Recent studies indicate that several proteins and cellular components, including $\alpha$-melanocyte-stimulating hormone, stem cell factors, ckit, vascular endothelial growth factor, and nerve growth factor, are involved in the pathogenesis of melasma.?

Topical agents such as hydroquinone and superficial chemical peels are the current standard for melasma treatment, but concern about side effects and long-term safety has spurred efforts to develop alternative treatment options. ${ }^{8}$ Oral intake or intralesional injection of ascorbic acid or tranexamic acid has been clinically tried. ${ }^{9}$ Lightbased treatment has been also suggested as a promising strategy for the management of melasma. While currently repeated irradiation with a 1,064 $\mathrm{nm}$ Q-switched $\mathrm{Nd}$ :YAG laser at low fluences is regarded as a standard laser treatment of melasma, ${ }^{10}$ newer approaches using different wavelengths and laser types have been investigated with variable success. ${ }^{11-16}$

Recently, the novel wavelength of 1,927 nm delivered by the thulium laser has been studied for the treatment of photoaging ${ }^{17}$ and melasma. ${ }^{18-20}$ This wavelength has a higher absorption coefficient for water than the conventional fractional 1,550 $\mathrm{nm}$ lasers, conferring a greater ability to target epidermal structures with precision with minimal damage to the dermis. The other characteristic of this wavelength that makes it well suited for superficial epidermal indications is its reported maximum depth of penetration of $200 \mu \mathrm{m}$. The present study investigates the safety and efficacy of the novel 1,927 nm thulium fiber laser system in the treatment of therapy-resisting melasma in Korean female subjects.

\section{MATERIALS AND METHODS}

A retrospective chart and photographic review of Korean female melasma patients treated with a novel 1,927 $\mathrm{nm}$ fractional thulium laser system from February 2015 to March 2016 was performed. All patients were clinically diagnosed as having melasma by a board-certified dermatologist (RNK). Only patients with moderate to severe melasma in whom previous therapies have failed were included for the review. Digital photographs of patients' frontal, oblique, and profile views were recorded using a 14.6 megapixel digital camera (Samsung NX-10 $0^{\mathrm{TM}}$; Samsung Electronics, Co., Ltd., Gyeonggi, Korea) under a constant light setting before treatment at each visit. Skin preparation was carefully performed with a gentle cleanser to remove debris and makeup before treatment. A cream containing a mixture of lidocaine $2.5 \%$ and prilocaine $2.5 \%$ was applied under occlusion for 20 minutes before laser treatments. The laser system used in this study was a novel 1,927 nm fractional thulium-doped fiber laser (LASEMD ${ }^{T M}$; Lutronic Co., Gyeonggi, Korea). Each shot with this laser system generates 40 fractionated zones of thermal injury over an area of about $10 \mathrm{~mm}$ $\times 4 \mathrm{~mm}$. There are three pre-set treatment levels: Level 1 corresponds to an output power of $3 \mathrm{~W}$ and a pulse energy of $1.6 \mathrm{~mJ}$; Level 2 corresponds to $4 \mathrm{~W}$ and $2 \mathrm{~mJ}$; and Level 3 corresponds to $5 \mathrm{~W}$ and $2.4 \mathrm{~mJ}$. After 3-5 full facial laser passes, manufacturer-supplied vitamin A (VA)-, vitamin C (VC)-, or tranexamic acid (TA)-containing hydrogel was applied to the treatment area. Post-treatment calming was performed using a light-emitting diode system (Smartlux ${ }^{\text {TM}}$; Medmix Co., Seoul, Korea) with a mixed 635 and $830 \mathrm{~nm}$ light and a power density of $75 \mathrm{~mW} / \mathrm{cm}^{2}$ for 15 minutes. Patients were instructed to avoid sun exposure 
Table 1. Melasma area and severity index $(\mathrm{MASI})$ score $=0.3(\mathrm{DF}+\mathrm{HF}) \mathrm{AF}+0.3(\mathrm{DMR}+\mathrm{HMR}) \mathrm{AMR}+0.3(\mathrm{DML}+\mathrm{HML}) \mathrm{AML}+0.1(\mathrm{DC}+$ $\mathrm{HC}) \mathrm{AC}$

\begin{tabular}{|c|c|c|c|c|c|c|c|}
\hline & 0 & 1 & 2 & 3 & 4 & 5 & 6 \\
\hline Darkness of pigment (D) & None & Slight & Moderate & Marked & Very marked & & \\
\hline Homogeneity of pigment $(\mathrm{H})$ & No pigment & Speckles & $<2 \mathrm{~cm}$ patches & $>2 \mathrm{~cm}$ patches & Homogenous & & \\
\hline Surface area involved $(A)$ & & $<10 \%$ & $10-29 \%$ & $30-49 \%$ & $50-69 \%$ & $70-89 \%$ & $90-100 \%$ \\
\hline Site involved & \multicolumn{2}{|c|}{ Forehead (F) } & \multicolumn{2}{|c|}{ Right malar (MR) } & \multicolumn{2}{|c|}{ Left malar (ML) } & Chin (C) \\
\hline
\end{tabular}

Table 2. Patient demographics

$\begin{array}{ll}\text { Number of patients } & 68 \\ \text { Sex } & 68 \text { females, 0 males } \\ \text { Age (years) } & 29-52 \text { (mean 43.9) } \\ \text { Fitzpatrick skin type } & \text { III, 41 (60.3\%) } \\ & \text { IV, 27 (39.7\%) } \\ \text { Melasma involvement area } & \text { Forehead/Temples, 47 (69.1\%) } \\ & \text { Cheeks, 68 (100\%) } \\ & \text { Chin, 4 (5.9\%) } \\ \text { Laser treatment } & \text { Level 2 (4W, 2 mJ), 55 (80.9\%) } \\ & \text { Level 3 (5W, 2.4 mJ), 13(19.1\%) } \\ \text { Adjunctive topical treatment } & \text { Vitamin A, 18 (26.5\%) } \\ & \text { Vitamin C, 30 (44.1\%) } \\ & \text { Tranexamic acid, 20 (29.4\%) }\end{array}$

and use topical VA, VC, or TA gel at home for 7 days after treatment. Three treatment sessions were performed with an interval of 2-4 weeks. Patients were followed up 2-4 weeks after the third treatment session.

Two independent investigators evaluated Melasma Area and Severity Index (MASI) scores (Table 1) based on digital photographs taken at each visit. The difference in MASI values before and after treatment was statistically analyzed using a paired Student's t-test (GraphPad Prism 7.0; GraphPad Software, Inc. CA, USA). Data are expressed as means standard errors, and $p<0.05$ was considered to be statistically significant. Patients were asked to assess their subjective satisfaction at each visit and during follow-up using a quartile grading scale: 1 , less than 25\% clearance; grade 2, 26-50\% clearance; grade $3,51-75 \%$ clearance; grade 4 , more than $75 \%$ clearance of melasma. The prevalence and nature of treatmentrelated adverse events, if any, were described. Melasma recurrence, defined as increase of pigment density or enlargement of involved area from the final visit, was evaluated for all patients who were successfully followed-up.

\section{RESULTS}

Patient demographic data are summarized in Table 2. All patients were Korean females, with a mean age of

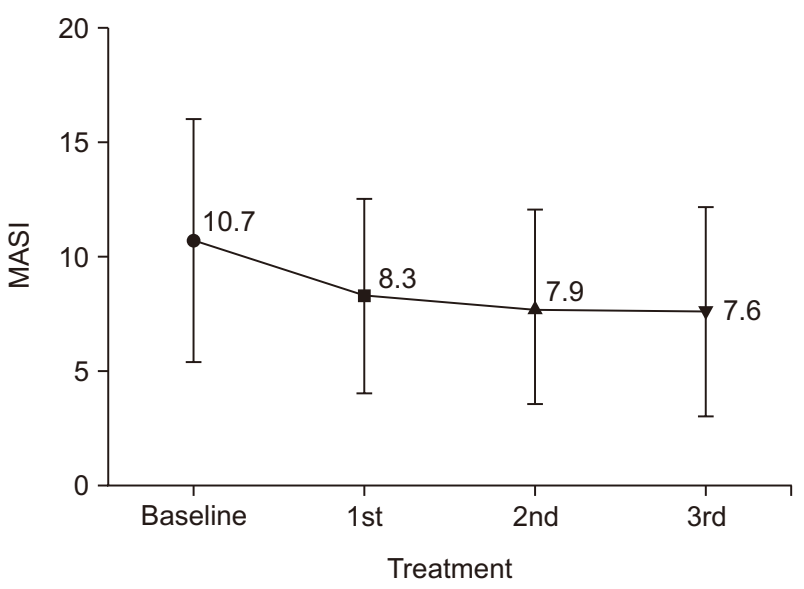

Fig. 1. Melasma Area and Severity Index (MASI) score changes according to serial treatments with the $1,927 \mathrm{~nm}$ fractional thulium laser.

43.9 years (range 29-52). Records of 204 consecutive laser treatment sessions in 68 patients were analyzed. Patient ages ranged from 29 to 52 years (mean 43.9). Subjects represented skin phototype either III or IV. Patients were treated with laser parameters of 4W-2 mJ (Level 2; 55 patients) or 5W-2.4 $\mathrm{mJ}$ (Level 3; 13 patients). Thirteen of the 68 patients (44.1\%) were adjunctively treated with topical VC gel. Topical VA (18 patients, 26.5\%) and TA (20 patients, $29.4 \%$ ) gels were used for post-laser treatment in 18 $(26.5 \%)$ and $20(29.4 \%)$ patients, respectively.

Objective photographic assessment found that the mean MASI scores decreased from $10.7 \pm 5.3$ at baseline to $7.6 \pm 4.6$ (29\% reduction) at 2 weeks after 3 consecutive laser treatment ( $p=0.0001$ ) (Fig. 1). A MASI score decrease of more than 3.0 was found in 34 patients (50.0\%). Three patients, all treated under a higher energy setting, showed an increase of their MASI scores despite undergoing laser therapy. Detailed MASI changes are shown in Fig. 2. The mean decrease from baseline in MASI scores was significantly greater for patients adjunctively treated with topical TA than VA or VC $(p=0.0091$, Kruskal-Wallis test; Fig. 3).

Patients' self-evaluation revealed a mean improvement 


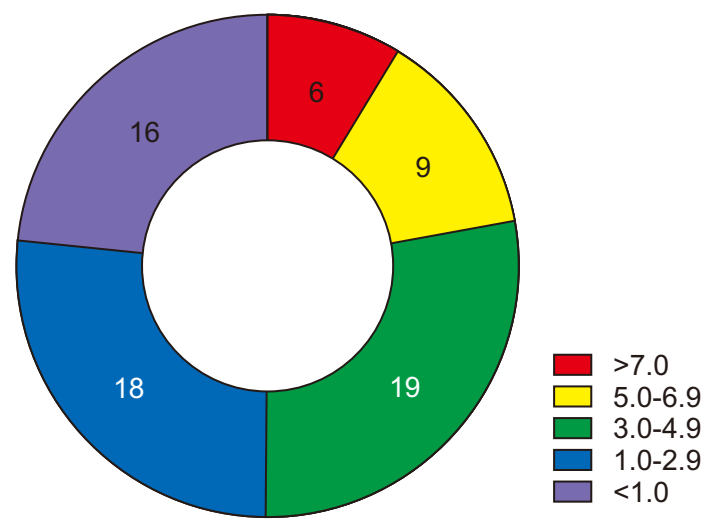

Fig. 2. MASI score decreases before and 2 weeks after 3 treatment sessions with the $1,927 \mathrm{~nm}$ fractional thulium laser.

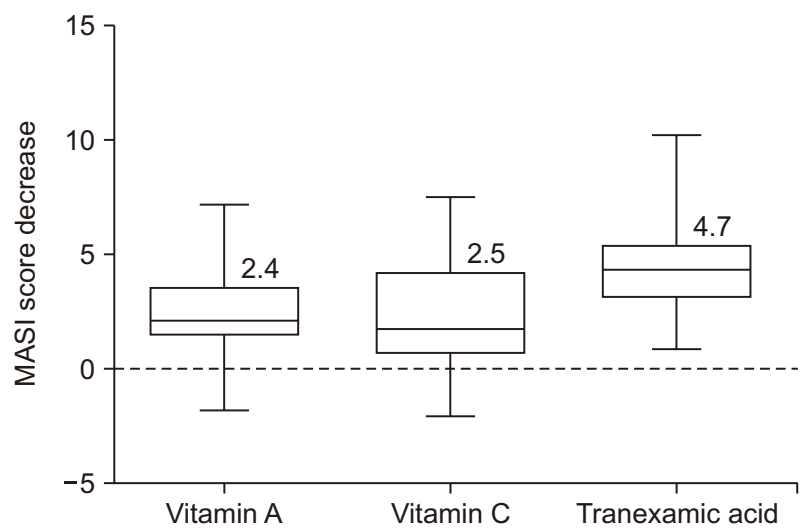

Fig. 3. Mean decrease of MASI scores after 1,927 nm fractional thulium laser treatment according to the post-laser topical care regimen.

grade of 2.4 , with 33 of 68 subjects (48.5\%) estimating more than $50 \%$ clearance of their melasma at 2 weeks after 3 laser treatments (Fig. 4). A representative before and after photograph is shown in Fig. 5. Other than melasma, patient-reported improved conditions included skin tone and texture (all patients), skin tightness (49 patients), decreased pore size ( 44 patients), and fine wrinkles (37 patients). Eight patients (8.8\%) reported that there was no or only minimal improvement (less than $25 \%$ ) of their melasma at the end of the treatment.

In most cases, post-treatment erythema and edema were mild and resolved spontaneously within 24-48 hours. Post-treatment skin dryness for 2-5 days was a common feature. Mild and transient PIH developed in three patients. Two patients who had pre-existing acne on the face experienced temporary post-laser flare of the acne lesions which did not require specific treatments. Recurrence of the melasma was observed in 13 patients

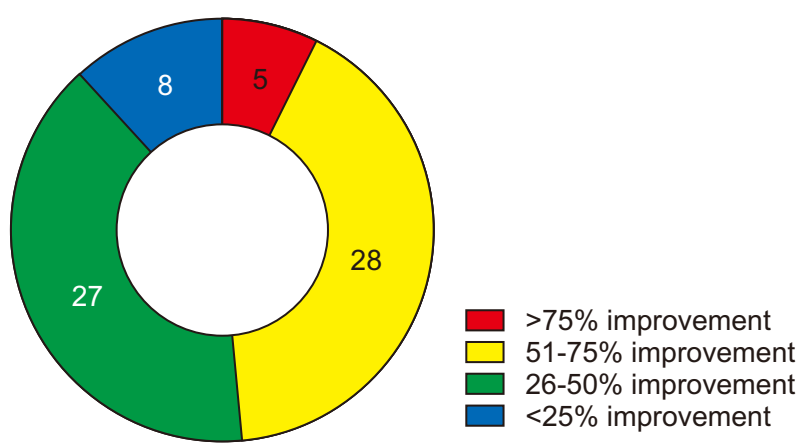

Fig. 4. Patients' self-evaluation of improvement of their melasma after the $1,927 \mathrm{~nm}$ fractional thulium laser.
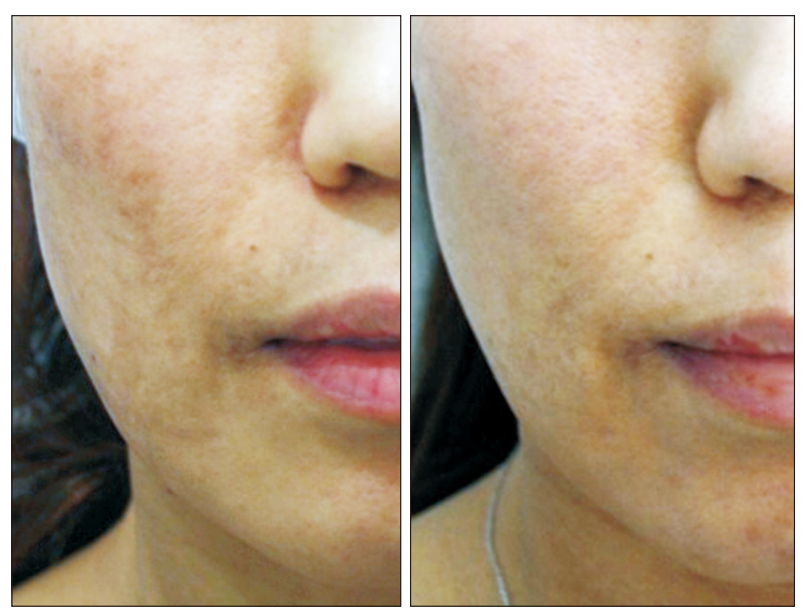

Fig. 5. Before and 2 weeks after 3 consecutive treatment sessions with the $1,927 \mathrm{~nm}$ fractional thulium laser ( $5 \mathrm{~W}, 2.4 \mathrm{~mJ}, 4$ passes).

out of 60 patients who were successfully followed-up for more than 2 months (2.0-7.8 months; mean 4.1).

\section{DISCUSSION}

The principle of melasma therapy is protection from sunlight and pigment reduction. Pigment reduction is achieved by using chemicals that interfere with various steps of the melanogenesis pathways via: (i) the retardation of abnromal melanocyte activity; (ii) the inhibition of melanosome formation and melanin synthesis; and (iii) the enhancement of melanosome degradation. ${ }^{21}$ Topical application of depigmenting agents, sometimes along with superficial chemical peels, is usually the first-line treatment. Laser and light therapies represent potentially promising options for patients who are refractory to other modalities. Because laser and light therapy frequently carry a significant risk of worsening the disease, a thorough understanding of the risks and benefits of each 


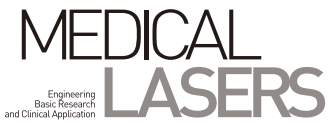

treatment option is crucial. ${ }^{21}$

Lasers and light sources have been evaluated in the treatment of melasma with varied results. ${ }^{14,16,21}$ The basic mechanism of action of laser and light-based treatment for the treatment of pigmentary disorders employs photothermal, photomechanical, or ablative effects. The principle of photothermolysis dictates that the target molecule, the chromophore, should preferentially absorb the delivered wavelength of light, and that the light energy must be delivered over a period of time to damage the target while limiting collateral damage to adjacent structures. ${ }^{16}$ Regarding melasma treatment, typical devices that rely on this principle include the low fluence 1,064 nm and 694 $\mathrm{nm}$ Q-switched lasers, which have been reported to have moderate clinical efficacy with minimal downtime and less side effects. ${ }^{10,12,16,21}$ Use of a long-pulsed laser with a pigment-selective wavelength has also been tried. ${ }^{15}$

Pigment non-specific fractional lasers have also been used to treat melasma. Fractional non-ablative resurfacing has been reported to be effective and safe for therapyresisting melasma. ${ }^{14,16}$ However due to its modest clinical efficacy, fractionated non-ablative laser treatment is regarded as an adjuvant therapy with topical bleaching creams. ${ }^{16}$ Another option of fractional laser treatment for melasma is ablative fractional resurfacing. The 2,940 $\mathrm{nm}$ erbium:YAG laser is ablative and highly absorbed by water reducing thermal skin damage, ${ }^{22}$ but its side effect profile is generally too high to be used for the treatment of melasma. ${ }^{16}$ When irradiated in a fractional pattern, this wavelength provides similar efficacy as traditional ablative approaches while avoiding their long downtime and severe side effects. ${ }^{11}$

The 1,927 nm fractionated non-ablative thulium laser targets epidermal pigment with greater efficacy than is seen with the 1,55 nm laser alone, due to its higher absorption coefficient for water. ${ }^{16,23}$ By targeting water as its chromophore, the laser induces a dense array of microscopic, columnar thermal zones of tissue injury, and subsequent "melanin shuttling" of underlying epidermal and dermal pigment coupled with remodeling of the pathologic dermis has been proposed as a possible mechanism. $^{24}$

Polder et al..$^{18}$ treated fourteen patients with melasma via a combined fractional $1,550 \mathrm{~nm}$ and $1,927 \mathrm{~nm}$ fractional thulium fiber laser. The patients underwent three to four laser treatments at 4 week intervals. After 3-4 treatments, a statistically significant $51 \%$ improvement in melasma was seen without any $\mathrm{PIH}$ at a 1-month follow-up and a 33\% and 34\% reduction at 3- and 6-month follow ups. Six months after the final laser treatment, melasma disease severity increased marginally, although patients were still improved from baseline. There were no statistically significant differences seen in MASI scoring between Fitzpatrick skin types I/II and III/IV. A retrospective chart and photographs review of 20 females with melasma ${ }^{19}$ confirmed the efficacy and safety of the 1,927 nm fractional laser alone in the treatment of melasma in patients with skin types II-IV. Mean MASI scores decreased from 13.2 before treatment to 8.5 at 4 weeks after treatment. Twelve of the 20 subjects reported more than $50 \%$ clearance of their melasma. Recurrence was reported by 7 out of 15 patients who were successfully followed-up and $\mathrm{PIH}$ was observed in 2 patients. The efficacy and safety of the fractional 1,927 nm laser was also evaluated in eleven Chinese patients with mild melasma, ${ }^{20}$ which showed significant MASI improvement early after one treatment. Significant improvement in skin texture and pore size was also observed. Main adverse effects were erythema, edema, and crusting in the early days. One patient developed $\mathrm{PIH}$.

In the present study, a statistically significant $29 \%$ reduction in the MASI score was observed after three consecutive 1,927 nm thulium fractional laser treatments, which is comparable to the result of the study by Massaki et al. ${ }^{19}$ (36\% reduction). It is noteworthy that the improvement of melasma was evident early after the first treatment in the present study. This tendency has also been described in previous trials. ${ }^{18-20}$ Considering that most melasma treatment modalities are related with slow clinical response and long-term, repeated applications, $8,10,14,21$ the quick therapeutic onset of the 1,927 nm fractional laser resurfacing can be a strong advantage in the treatment of melasma. This implies that early clinical improvement of melasma can first be achieved by ablative fractional laser resurfacing and other treatment modalities can be applied after it for maintenance.

One major difficulty in the treatment of melasma is the high rate of recurrence associated with any of the therapeutic options. ${ }^{19}$ In the present study, the recurrence rate was $21.7 \%$ in sixty patients who were successfully followed-up for a mean of 4 months. This is lower than that of the report of Massaki et al. ${ }^{19}$ (7 out of 15, mean follow-up 10 months). When the relatively short duration of follow-up in the present study is taken into consideration, the actual recurrence rate is expected to be higher. The etiologic factors of melasma usually persist following laser therapy, thus higher recurrences are inescapable. Combination therapies in the treatment of melasma should be pursed to achieve better results. ${ }^{16}$

$\mathrm{PIH}$ developed in three patients and it was mild in de- 
gree and transient in duration. Of interest is the low rate of such complications in the present study and previous two reports ${ }^{19,20}$ since PIH remains a serious concern of fractional thermolysis in dark-skinned patients. ${ }^{20}$ The relatively low rate of $\mathrm{PIH}$ development in fractional 1,927 nm laser resurfacing can be due to the low penetration depth of the 1,927 nm wavelength laser. In the present study, heat damage could be expected to be confined to the epidermis in a fractionated pattern and this can explain the low rate of $\mathrm{PIH}$ since $\mathrm{PIH}$ is associated with the disruption of the dermoepidermal junction and inflammation in the upper dermis. ${ }^{25}$ The thulium laser system used in the study has been described to create coagulation columns confined to the epidermis at low energy settings and be capable of creating coagulation zones extending from the epidermis through the upper dermis at higher power and pulse energy settings, ${ }^{23}$ which clearly indicates that the depth of the 1,927 nm fractional laser treatment zone is determined by the energy setting. In melasma, the target pigments are located in the epidermis and a more conservative energy setting is suggested to adequately target epidermal pigments. ${ }^{20}$ High energy settings along with increased surface area coverage should be avoided to minimize the risk of $\mathrm{PIH}$ when treating melasma patients with darker skin types.

Development of hypopigmented macules is one of the serious complication of conventional Q-switched laser treatment of melasma. ${ }^{10,12}$ Until now, no case of hypopigmentation has been reported in melasma patients treated with the 1,927 nm laser. Two patients experienced worsening of pre-existing acne in the present study. Transient acne flare-up is common after fractional laser resurfacing and it is suggested to be associated with disruption of the pilosebaceous units during laser treatment and subsequent aberrant follicular epithelialization during healing. ${ }^{26}$

One limitation of the present study was that it involved patients with moderate to severe melasma (mean baseline MASI score of 11.7) only. The efficacy and safety of the 1,927 $\mathrm{nm}$ fractional laser on clinically mild and limited types of melasma should be evaluated further.

In conclusion, the present study demonstrates the efficacy and safety of a novel 1,927 nm fractional thulium laser in Korean patients with moderate to severe melasma resistant to conventional therapies. Conservative energy settings with repeated treatments are recommended to minimize PIH and lower recurrence rates.

\section{REFERENCES}

1. Newcomer VD, Lindberg MC, Sternberg TH. A melanosis of the face ("chloasma"). Arch Dermatol 1961;83:284-99.

2. Handel AC, Miot LD, Miot HA. Melasma: a clinical and epidemiological review. An Bras Dermatol 2014;89:771-82.

3. Kang WH, Yoon KH, Lee ES, Kim J, Lee KB, Yim H, et al. Melasma: histopathological characteristics in 56 Korean patients. Br J Dermatol 2002;146:228-37.

4. Lee MH, Noh TK, Lee JH, Roh MR, Na Jl, Jung EC, et al. Clinicoepidemiological features of melasma in Korean patients at five university hospitals: a cross-sectional multicenter study. Korean J Dermatol 2016;54:532-7.

5. Kwon SH, Hwang YJ, Lee SK, Park KC. Heterogeneous pathology of melasma and its clinical implications. Int J Mol Sci 2016;17:E824.

6. Hernández-Barrera R, Torres-Alvarez B, Castanedo-Cazares JP, Oros-Ovalle C, Moncada B. Solar elastosis and presence of mast cells as key features in the pathogenesis of melasma. Clin Exp Dermatol 2008;33:305-8.

7. Chung BY, Noh TK, Yang SH, Kim IH, Lee MW, Yoon TJ, et al. Gene expression profiling in melasma in Korean women. Dermatology 2014;229:333-42.

8. Ball Arefiev KL, Hantash BM. Advances in the treatment of melasma: a review of the recent literature. Dermatol Surg 2012;38:971-84.

9. Tse TW, Hui E. Tranexamic acid: an important adjuvant in the treatment of melasma. J Cosmet Dermatol 2013;12:57-66.

10. Cho SB, Kim JS, Kim MJ. Melasma treatment in Korean women using a 1064-nm Q-switched Nd:YAG laser with low pulse energy. Clin Exp Dermatol 2009;34:e847-50.

11. Lapidoth M, Yagima Odo ME, Odo LM. Novel use of erbium:YAG (2,940-nm) laser for fractional ablative photothermolysis in the treatment of photodamaged facial skin: a pilot study. Dermatol Surg 2008;34:1048-53.

12. Jang WS, Lee CK, Kim BJ, Kim MN. Efficacy of 694-nm Q-switched ruby fractional laser treatment of melasma in female Korean patients. Dermatol Surg 2011;37:1133-40.

13. Zaleski L, Fabi S, Goldman MP. Treatment of melasma and the use of intense pulsed light: a review. J Drugs Dermatol 2012;11:1316-20.

14. Rivas S, Pandya AG. Treatment of melasma with topical agents, peels and lasers: an evidence-based review. Am J Clin Dermatol 2013;14:359-76.

15. Lee MK, Min KS, Park EJ, Kim KH, Kim KJ. A retrospective analysis of the treatment of melasma using a fractional longpulsed alexandrite laser in Korean patients. Dermatol Surg 2016;42:952-60.

16. Dunbar S, Posnick D, Bloom B, Elias C, Zito P, Goldberg DJ. Energy-based device treatment of melasma: an update and 
review of the literature. J Cosmet Laser Ther 2017;19:2-12.

17. Lee HM, Haw S, Kim JK, Chang SE, Lee MW. Split-face study using a 1,927-nm thulium fiber fractional laser to treat photoaging and melasma in Asian skin. Dermatol Surg 2013;39:879-88.

18. Polder KD, Bruce S. Treatment of melasma using a novel 1,927-nm fractional thulium fiber laser: a pilot study. Dermatol Surg 2012;38:199-206.

19. Niwa Massaki AB, Eimpunth S, Fabi SG, Guiha I, Groff W, Fitzpatrick R. Treatment of melasma with the 1,927-nm fractional thulium fiber laser: a retrospective analysis of 20 cases with long-term follow-up. Lasers Surg Med 2013;45:95101.

20. Ho SG, Yeung CK, Chan NP, Shek SY, Chan HH. A retrospective study of the management of Chinese melasma patients using a $1927 \mathrm{~nm}$ fractional thulium fiber laser. J Cosmet Laser Ther 2013;15:200-6.

21. Shankar K, Godse K, Aurangabadkar S, Lahiri K, Mysore V,
Ganjoo A, et al. Evidence-based treatment for melasma: expert opinion and a review. Dermatol Ther (Heidelb) 2014;4:165-86.

22. Manaloto RM, Alster T. Erbium:YAG laser resurfacing for refractory melasma. Dermatol Surg 1999;25:121-3.

23. Cho SB, Zheng Z, Kang JS, Kim HS. Therapeutic efficacy of 1,927-nm fractionated thulium laser energy and polydeoxyribonucleotide on pattern hair loss. Med Lasers 2016;5:22-8.

24. Hantash BM, Bedi VP, Sudireddy V, Struck SK, Herron GS, Chan KF. Laser-induced transepidermal elimination of dermal content by fractional photothermolysis. J Biomed Opt 2006;11:041115.

25. Goldberg DJ, Berlin AL, Phelps R. Histologic and ultrastructural analysis of melasma after fractional resurfacing. Lasers Surg Med 2008;40:134-8.

26. Rho NK. Short- and long-term side effects of non-ablative fractional laser resurfacing in Koreans: analysis of 286 consecutive treatments. Med Lasers 2014;3:17-21. 